NASA Technícal Memorandum 100841

$-1$

\title{
Optical RF Distribution Links for MMIC Phased Array Antennas
}

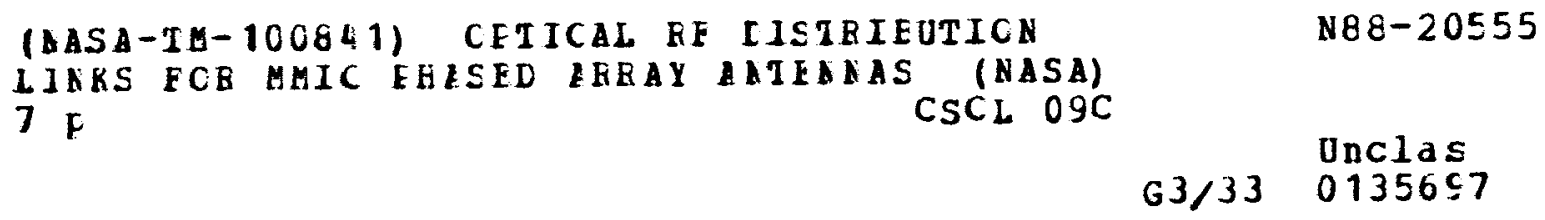

Richard R. Kunath, Kul B. Bhasin, and Charles A. Raquet

Lewis Research Center

Cleveland, Ohio

Prepared for the 1987 IEEE AP-S International Symposium and URSI Radio Science Meeting

Blacksburg, Virginia, June 15-19, 1987 


\title{
OPTICAL RF DISTRIBUTION LINKS FOR MMIC PHASED ARRAY ANTENNAS
}

\author{
Richard R. Kunath, Kul B. Bhasin, and Charles A. Raquet \\ National Aeronautics and Space Administration \\ Lewis Research Center \\ Cleveland, Ohio 44135
}

\section{SUMMARY}

Conventional methods to distribute RF signals to GaAs Monolithic Microwave Integrated Circuit Phased Array Antennas are inadequate for arrays having large numbers of elements. Optical RF distribution links have been proposed as a lightweight, mechanically flexible, and low volume solution. Three candidate techniques for providing optical RF distribution are discussed along with the electro-optic devices required to configure them. A discussion of the present status of applicable electro-optics devices is also included.

\section{INTRODUCTION}

As GaAs Monolithic Microwave Integrated Circuits (MMICs) become more prevalent, they are increasingly being used in real applications as key elements for amplitude and phase control in phased array antennas. As experience grows with the construction of these arrays, it is apparent that the present methods of RF signal distribution have serious complications which greatly impact the array construction. Of primary concern is the weight of the Beam Forming Network (BFN) using conventional coaxial cable or waveguide. Also impacting design is the loss, cross-talk, and mechanical inflexibility of current distribution media including microstrip. A potential solution to the se limitations is the use of optical fiber as the RF distribution medium in the BFN (ref. 1). In addition to the significant advantages over other RF distribution media, optical fiber offers certain EMI, EMP, and radiation hardening characteristics. This paper will outline and describe NASA Lewis Research Center's efforts in the development of optical RF distribution links for MMIC phased array antennas.

\section{Optical RF Distribution Architectures}

Three candidate architectures are being investigated for performance and eventual use in operational MMIC-based phased arrays. The three architectures differ in the modulation of RF and communications data. One architecture uses a direct modulation technique, a second architecture uses an indirect (external) modulation technique, and the third architecture uses an injection locking technique with mixing. Each architecture will be investigated experimentally in a distribution involving at least two elements. Prior to testing, the power budget will be calculated using a computer simulation model developed at Drexel University and compared against real data (ref. 2). Figure 1 shows a directly modulated RF distribution system for two elements. The master oscillator operated at $18 \mathrm{GHz}$ is modulated by a $200 \mathrm{Mbs}$ pseudo-random generated data stream. $18 \mathrm{GHz}$ is chosen as the RF carrier because it is planned to use the $20 \mathrm{GHz}$ MMICs developed for NASA by Texas Instruments and Rockwell International. The device bandwidth is 17.5 to $20.0 \mathrm{GHz}$. These devices are described in detail in the references (ref. 3 ). 
Figure 2 is a four-element RF distribution link that uses external modulation techniques. As in all three models, the externally modulated link will use GaAs laser diodes as sources, lasing at approximately 0.8 m wavelengths. The modulator itself will be of the $\mathrm{LiNbO}_{3}$ variety which has been previously demonstrated at $17 \mathrm{GHz}$, but modulation frequencies may be increased by various improvements.

Figure 3 illustrates a two-element RF distribution link using an injection locking technique demonstrated by Drexel University (ref. 4). While more complex than the previous architectures, this technique should offer a high frequency, narrow bandwidth link with lower noise and greater dynamic range. This technique takes advantage of the inherent nonlinearity of the laser diode to utilize harmonic injection locking of free-running element microwave oscillators from a single master oscillator, to establish temporal coherence. The data to be transmitted is routed through a second low-speed ( $1 \mathrm{GHz}$ ) link. At the element, the RF and data are mixed to produce the desired output.

Ali candidate architectures will be analyzed for power budgets, S/N, biterror-rate (BER) and efficiency. The experimental set-up for BER testing is similar to that used at NASA Lewis for TWT evaluation and is detailed in the references (ref. 5 ).

Devices and Components for Direct/Indirect RF Distribution Links

Direct laser modulation at $0.8 \mu \mathrm{m}$ wavelengths using GaAs/GaAlAs semiconductor lasers has been demonstrated up to $10 \mathrm{GHz}$ (ref. 6). Bandwidths up to $16 \mathrm{GHz}$ at $20{ }^{\circ} \mathrm{C}$ and $26.5 \mathrm{GHz}$ at $-60{ }^{\circ} \mathrm{C}$ in a $1.3 \mu \mathrm{m}$ InGaAsP laser have also been achieved (ref. 7 ).

Indirect or external modulation has been demonstrated at frequencies up to $17 \mathrm{GHz}$ by using $\mathrm{LiNbO}_{3}$ crystals (ref. 8). The frequency range can be further extended by various improvements. The threshold for optical power damage and the efficiency for integrated optical modulators is low. If such modulators are designed on GaAs, they offer the possibility of monolithic integration.

The insertion loss parameters for the RF link have been obtained by Stephens, et al. (ref. 9) and are given below:

$$
\text { Insertion Los }=E_{m} \frac{R_{S}}{R_{L}}\left(n_{L} R_{D} \alpha_{0}\right)^{-2}
$$

where $R_{L}$ and $R_{S}$ are the load and source impedances, $n_{L}$ is the laser efficiency, $R_{D}$ is the detector responsivity, $\alpha_{0}$ is the optical loss, and $E_{m}$ is the matching circuit loss. It has been calculated, that by using optimized values of the parameters in equation (1), the intrinsic insertion loss of the electrical optical conversion could be reduced to a minimum of $10 \mathrm{~dB}$.

The overall S/N for a single optical fiber link depends on the photodetector shot and thermal noise together with the thermal noise associated with the amplifier following the photodetector, as well as the laser intensity noise. For signal transmission at GHz frequencies, laser noise is generally dominant. Photodetectors with a wide bandwidth are essential. An InGaAs/InP 
photodetector has been grown on a GaAs substrate for detecting wide bandwidth RF signals from $1.3 \mu \mathrm{m}$ InGaAsP lasers (ref. 10).

If such optical RF fiber links are viable, lasers and photodetectors can be monolithically integrated to reduce weight, power, cost and circuit parasitics. For a MMIC-based phased array signal distribution network, a number of Optical Integrated Circuits (OICS) will be required. For example, for the input RF signal a high frequency external modulator on GaAs with high efficiency and high optical damage threshold is needed as well as wide-band photodetectors. Figure 4 shows an RF signal OIC integrated with a $20 \mathrm{GHz} \mathrm{MMIC}$ Variable Phase Shifter. Additionally, a high-power integrated laser capable of being directly or indirectly modulated at high frequencies with an integrated driver will be required.

\section{CONCLUSION}

Three candidate architectures have been shown for optical RF distribution in microwave phased arrays. Each architecture is characterized by the modulation technique used. The overall array design is driven by both the architecture and the devices. However, once the architecture is chosen, the design is driven by the capability of the devices to meet the architecture requirements. The evaluation of these three candidate architectures with simulated communications information will determine which design should be optimized for an operational phased array antenna system and also will identify critical device technologies.

\section{REFERENCES}

1. K.B. Bhasin, G. Anzic, R.R. Kunath, and D.J. Connolly, "Optical Techniques To Feed And Control GaAs MMIC Modules For Phased Array Antenna Applications," AIAA 11 th Communications Satellite Systems Conference, New York: AIAA, 1986, pp. 506-514. (NASA TM-87218.)

2. P.R. Herczfeld, M. Kam, N. Laprade, K.B. Bhasin, and R.R. Kunath, "System Architecture for Large Aperture Space - Based Phased Array Antennas," Optical Technologies for Space Communication Systems, SPIE Vol. 756, K. Bhasin and G.A. Koepf, eds., Bellingham, WA: SPIE, 1987, pp. 164-172.

3. K.B. Bhasin and D.C. Connolly, "Advances in Gallium Arsenide Monolithic Microwave Integrated Circuit Technology for Space Communications Systems," IEEE Trans. Microwave Theory Technol., vol. MTT-34, pp. 994-1001, 1986.

4. A. Daryoush, P. Herczfeld, V. Contarino, A. Rosen, Z. Turski, and P. Wahl, "Optical Beam Control of Millimeter Wave Phased Array Antennas For Communications," 16th European Microwave Conference, Microwave Exhibitions and Publishers Ltd., Kent, England: 1986.

5. K.A. Shalkhauser and G. Fujikawa, "Bit-Error-Rate Testing Of High-Power $30 \mathrm{GHz}$ Traveling Wave Tubes For Ground-Terminal Applications, "NASA TP-2635, 1986. 
6. K.Y. Lau, C. Harder, and A. Yariv, "Direct Modulation Of Semiconductor Lasers at $f>10 \mathrm{GHz}$ by Low-Temperature Operation," Appl. Phys. Lett., vol. 44, pp. 273-275, 1984.

7. K.Y. Lau, N. Bar-Chaim, I. Ury, C. Harder, and A. Yariv, "Direct Amplitude Modulation of Short-Cavity GaAs Lasers Up To X-Band Frequencies, "Appl. Phys. Lett., vol. 43, pp. 1-3, 1983.

8. H. Blauvelt and H. Yen, "Transmitter And Receiver Design For Microwave Fiber Optic Links," Optical Technology For Microwave Applications, SPIE Vol. 477, S.K. Yao, ed., Bellingham, WA: SPIE, 1984, pp. 44-51.

9. T.R. Joseph, W.E. Stephens, and B.U. Chen, "Fiber Optic RF Links," Optical Technology for Microwave Applications, SPIE Vol. 477, S.K. Yao, ed., Bellingham, WA: SPIE, 1984, pp. 52-56.

10. J.E. Bowers, C.A. Burrus, and R.J. McCoy, "InGaAs PIN Photodetectors With Modulation Response To Millimeter Wavelengths, "Elect. Lett., vol. 21, pp. 812-814, 1985 .

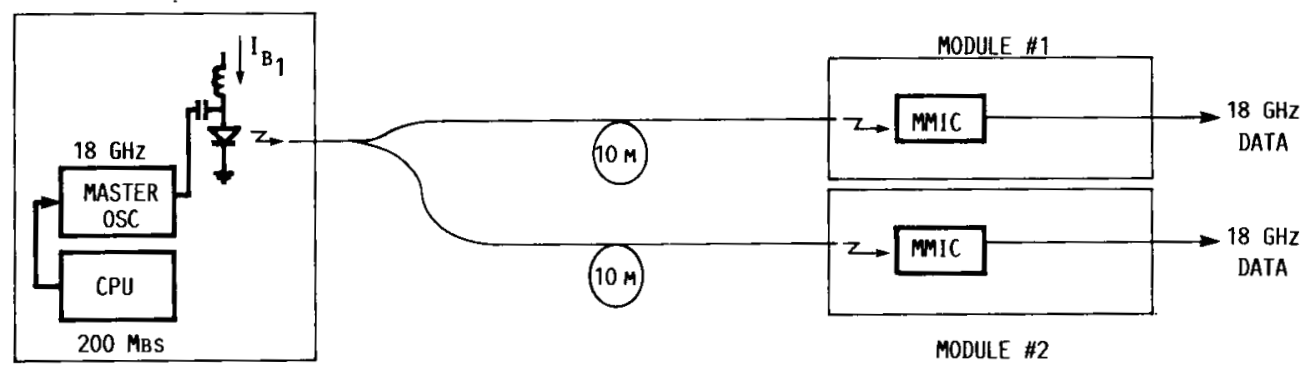

FIGURE 1. - DIRECTLY MODULATED RF/DATA LINK.

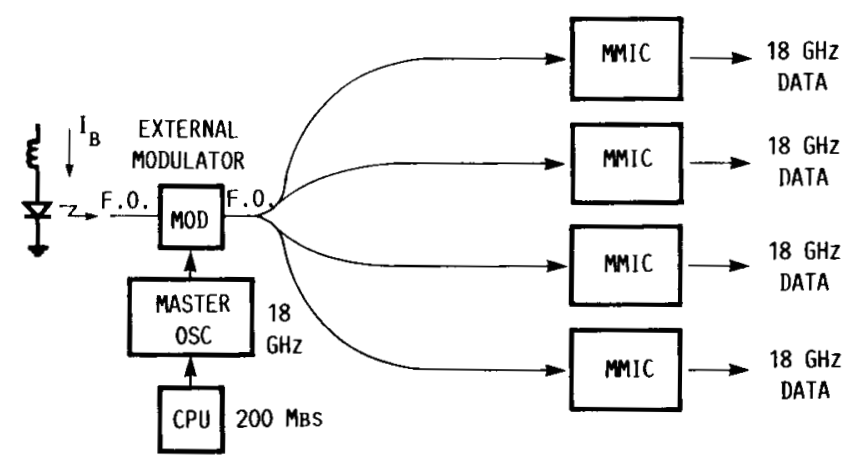

FIGURE 2. - EXTERNALLY MODULATED RF/DATA LINK. 


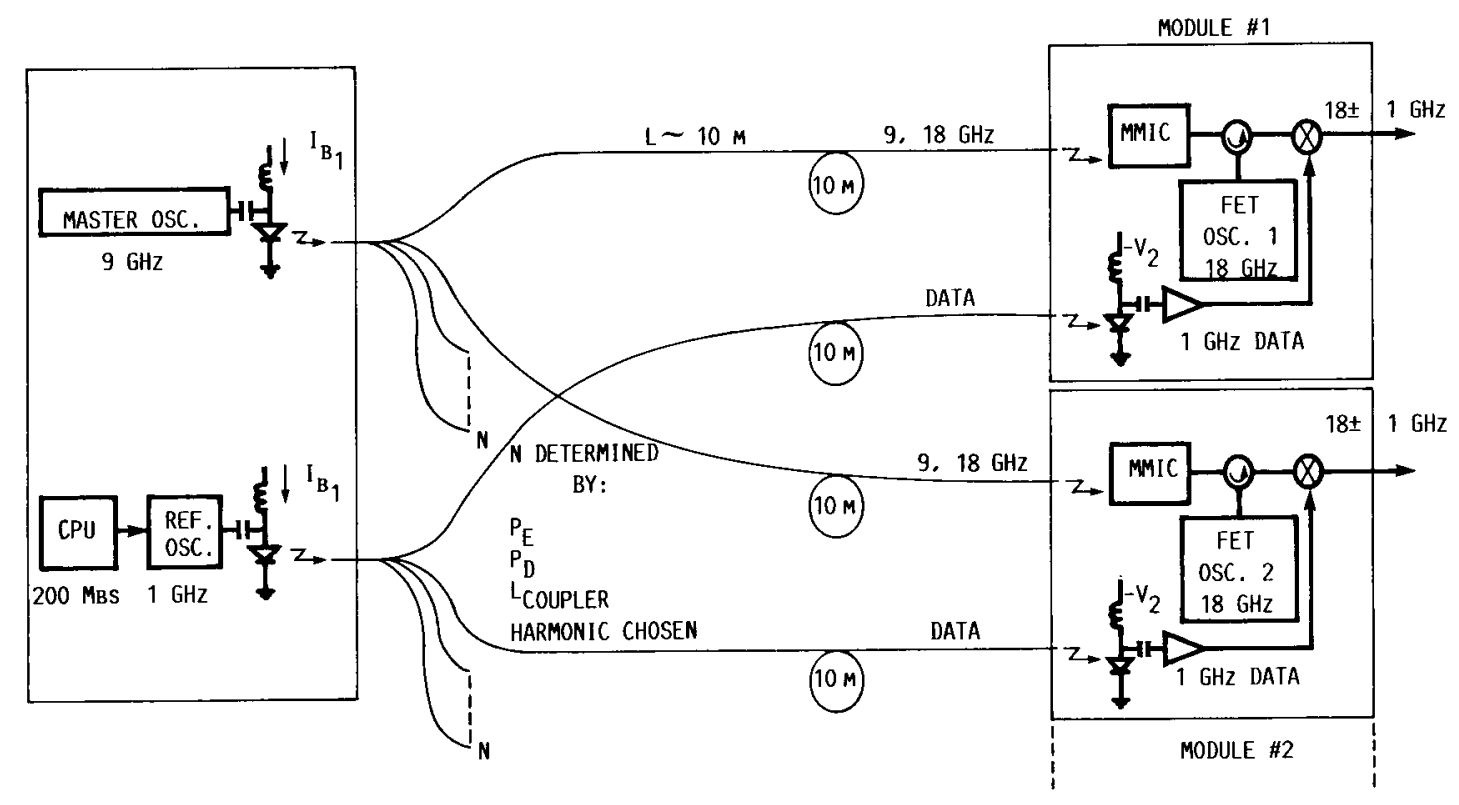

FIGURE 3. - INJECTION LOCKED RF/DATA LINK.

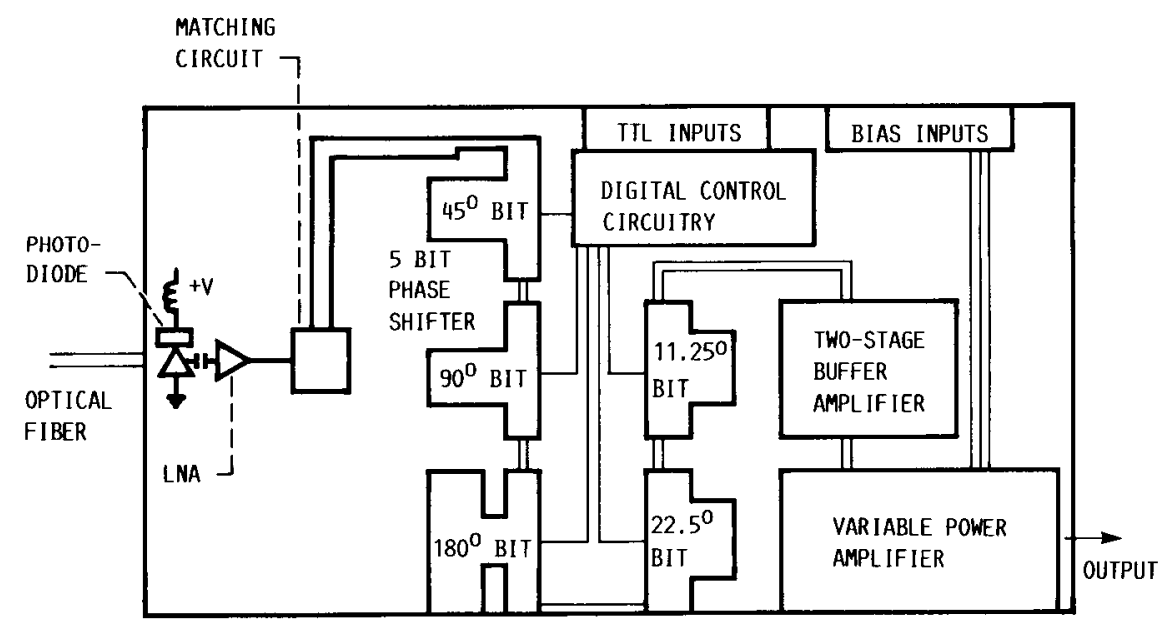

FIGURE 4. - $20 \mathrm{GHz}$ GaAs TRANSMIT MODULE WITH WIDE-BAND INTEGRATED PHOTODIODE. 


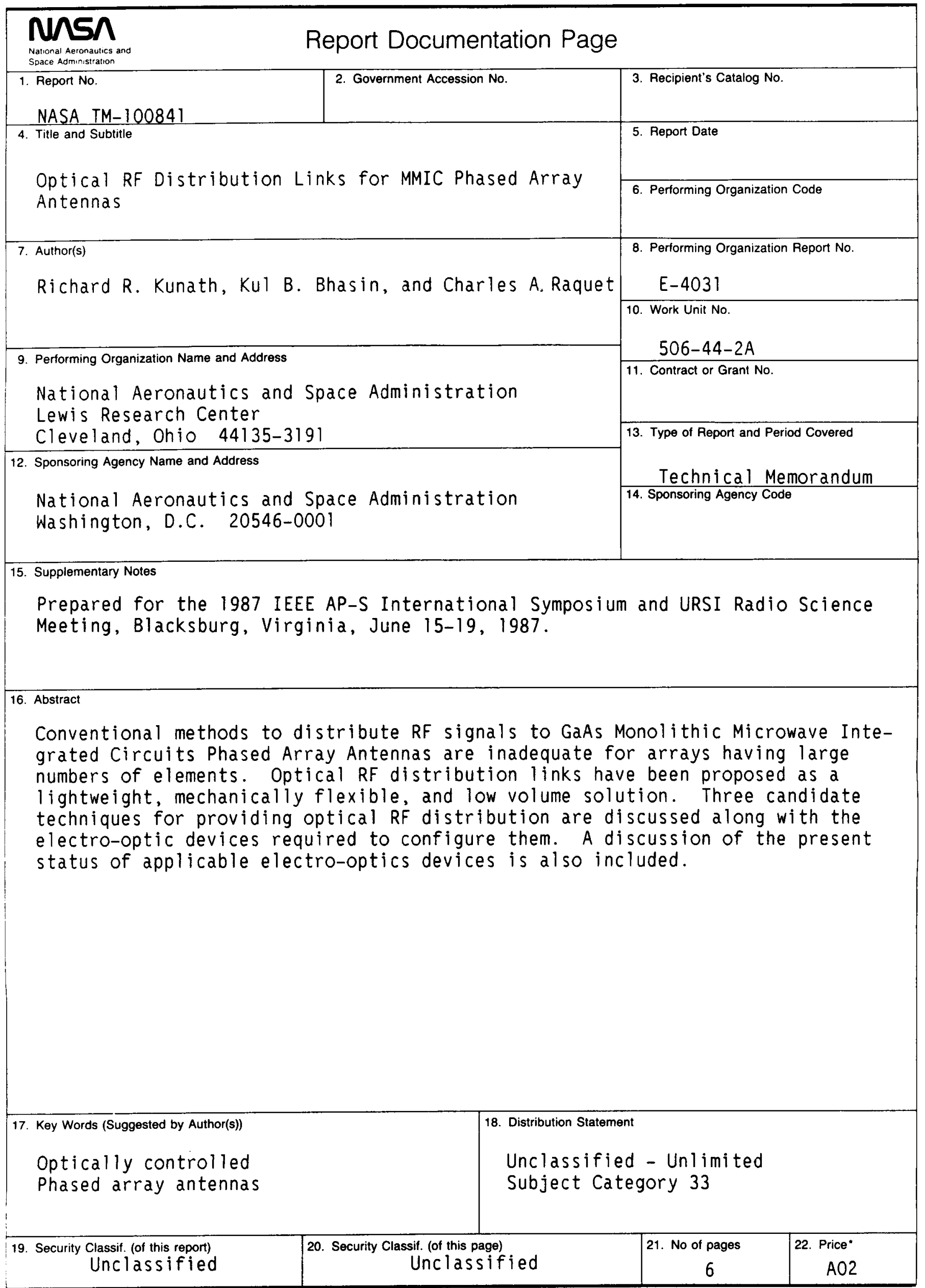

